\title{
Thrombolyse nicht wirksamer als Placebo
}

Fragestellung: Ist bei intraventrikulären Blutungen die Thrombolyse mit rt-PA über einen Ventrikelkatheter wirksam?

Hintergrund: Die Sterblichkeit bei intraventrikulären Blutungen beträgt $>50 \%$. Weniger als $20 \%$ der Überlebenden haben einen guten Outcome. Daniel Hanley aus Baltimore entwickelte eine neue Methode, bei der über einen Ventrikelkatheter ein Gewebeplasminogenaktivator appliziert und dann das verflüssigte Blut aspiriert wird.

Patienten und Methodik: Die randomisierte doppelblinde CLEAR-III-Studie schloss Pa-

Hanley DF, Lane K, McBee N et al. Thrombolytic removal of intraventricular haemorrhage in treatment of severe stroke: results of the randomised, multicentre, multiregion, placebocontrolled CLEAR III trial. Lancet 2017; 389: $603-11$ tienten mit einer nicht traumatischen intrazerebralen Blutung von $<30 \mathrm{ml}$ und intraventrikulärer Blutung ein. Über einen Ventrikelkatheter wurde bis zu zwölfmal physiologische Kochsalzlösung oder $1 \mathrm{mg}$ Alteplase alle acht Stunden appliziert. CT-Kon- trollen erfolgten während der Behandlung alle 24 Stunden. Der primäre Endpunkt war ein guter funktioneller Outcome, gemessen mit der modifizierten Rankin-Skala mit einem Wert zwischen 0 und 3 nach 180 Tagen.

Ergebnisse: Es wurden 500 Patienten randomisiert. Der primäre Endpunkt war mit $48 \%$ in der Alteplase- und $45 \%$ in der Placebogruppe identisch. Ein kleiner Therapieeffekt fand sich bei $\mathrm{Pa}$ tienten mit höherem Blutvolumen in den Ventrikeln und einer Blutung im Thalamus. Die Sterblichkeit war nach 180 Tagen mit $18 \%$ versus $29 \%$ (Hazard Ratio 0,60) signifikant zugunsten von rt-PA reduziert. Allerdings verblieben auf diese Weise mehr Patienten mit schwerer Behinderung in der aktiven Therapiegruppe. Eine Ventrikulitis trat unter rt-PA seltener auf als unter Kochsalzlösung. Schwerwiegende Nebenwirkungen waren mit $56 \%$ versus $60 \%$ in der rt-PA-Gruppe ebenfalls geringer. Die Rate symptomatischer Blutungen war mit $2 \%$ versus $1,2 \%$ identisch.

Schlussfolgerungen: Bei Patienten mit intraventrikulärer Blutung ist eine Thrombolyse mit rt-PA nicht besser wirksam als eine Placebobehandlung mit physiologischer Kochsalzlösung.

\section{- Kommentar von Hans-Christoph Diener, Essen}

\section{Diese Therapie wird Einzelfällen vorbehalten bleiben}

Diese große Studie zeigt die erheblichen Schwierigkeiten, zerebrale Blutungen zu behandeln. Der funktionelle Outcome war bei aktiver Behandlung und bei Placebobehandlung identisch. Die reduzierte Sterblichkeit unter rt-PA führte zu einer signifikanten Zunahme von Patienten mit schwersten neuro- logischen Ausfällen, ein Therapieergebnis, was definitiv nicht erwünscht ist. Leider gab es keine Untergruppe innerhalb der Studie, die von einer Thrombolyse bei intraventrikulärer Blutung wirklich profitiert hätte. Im klinischen Alltag wird diese Therapie daher Einzelfällen vorbehalten bleiben.

\section{Antiepileptika erfolgreich absetzen}

Fragestellung: Ermöglicht eine Metaanalyse eine Verbesserung der Beratung anfallsfreier Patienten hinsichtlich des Absetzens von Antiepileptika?

Hintergrund: Ein Nachteil von Studien ist das Fehlen evidenzbasierter Erfolgsprädiktoren sowie von Hilfestellungen für die individualisierte Einschätzung.

Patienten und Methodik: Es handelt sich um eine Metaanalyse von englischsprachigen Studien, die bis zum Jahr 2014 durchgeführt wurden. Epilepsiechirurgische Kohorten sowie Arbeiten mit weniger als 30 Patienten oder mit Patienten mit akuten symptomatischen (provozierten) Anfällen waren ausgeschlossen. Die
Analysen basierten auf den individuellen Daten der Teilnehmer von insgesamt zehn Studien und bezogen sich sowohl auf das generelle Rückfallrisiko nach zwei und nach fünf Jahren als auch auf die Chance einer mindestens einjährigen Anfallsfreiheit zehn Jahre nach dem Absetzversuch, unabhängig davon, ob wegen Anfallsrezidiven erneut eine Antiepileptikatherapie erfolgte. Überlebenskurven und proportionale Risiken wurden berechnet und mithilfe einer Rückwärtsselektion die aussagekräftigsten acht Prädiktoren ermittelt. Schließlich wurden Nomogramme zur Ermittlung der individuellen Erfolgschancen umgesetzt.

Ergebnisse: Von zunächst 45 Studien mit 7.082 Patienten konnten letztlich nur zehn mit 1.769 Patienten berücksichtigt werden 